\title{
Treatment of Recurrent Anterior Dislocations of Shoulder by Laterjet-Bristow Operation: An Experience
}

\author{
Bajracharya $A R^{1}$ Anjum $M P^{2}$ \\ 'Department of Orthopaedics, Bir hospital and Capital Hospital, Kathmandu, Nepal. \\ ${ }^{2}$ Dow university of Health Sciences and Civil hospital, Karachi, Pakistan.
}

\begin{abstract}
Shoulder dislocation is common problem in orthopaedics. Management of recurrent shoulder dislocation is painstaking with different surgical procedures having varying outcomes. LaterjetBristow procedure provides stability and good functional outcome in Rowe scale and long term patient satisfaction.
\end{abstract}

This is an observational prospective study carried out at two different tertiary care centers at Nepal and Pakistan using the same protocol. Habitual and pathological recurrent dislocations were excluded and all the recurrent dislocation of shoulder following initial traumatic anterior dislocation was included in this study. Standard Laterjet-Bristow operation was done and followed up for average of 27 months. Functional evaluation was done using Rowe score and graded as excellent, good, fair and poor.

There were 12 men and 9 women with the mean ages of 23.4 years. At 12 months, 11 patients had fair result and seven patients had good result. At two years only three had fair result, 15 had good result and three patients had excellent result. Only three patients had poor result at one year while none had poor result at two year. The mean loss of external rotation in operated shoulder at one year was 24.33 degrees which decreased to 21.2 degrees at the end of second year. There was no restriction of external rotation in three patients at both yearly follow-ups while 18 patients had restriction in the range 10-30 degrees.

Laterjet-Bristow can be the procedure of choice for surgical treatment of recurrent traumatic anterior dislocation and also as a salvage surgery for failed cases from other types of procedures. The only shortcoming of this procedure was some limitation in external rotation and minor loss in muscle power of that shoulder.

Key words: Laterjet-Bristow, Recurrent shoulder dislocation, Rowe scale.

\author{
Correspondence: \\ Dr. Ashok Raj Bajracharya \\ Department of Orthopaedics \\ Bir Hospital, NAMS, Kathmandu, Nepal. \\ Email: ashok_baj@hotmail.com
}




\section{INTRODUCTION}

Recurrence of dislocation in shoulder reaches up to 80 $90 \%$ in young adults and are disabling. Surgery is the treatment of choice. More than 150 operations has been described. Some how for fear of screw related technical difficulty and complications, Bristow procedure is not so popular. However our experience with this procedure in comparision with other popular simpler procedures in our practice had been superior in Rowe scale.

Transfer of the tip of the coracoid process to the anterior margin of the glenoid was first described by Laterjet for treatment of recurrent dislocation of the shoulder. ${ }^{1}$ Helfet used sutures to hold the coracoid tip. ${ }^{2}$ Mead screwed the coracoid to the anterior glenoid rim. ${ }^{3}$

There are several reports on the Laterjet-Bristow procedure and its modification. ${ }^{1,4}$ All of them reported low incidences of redislocations and other complications but many reported about limitation in external rotation. A 20 year follow up study from Middlesex Hospital, London, showed that it provided good long-term shoulder stability. .,6 $^{2}$ Hence this prospective study was carried out at two centres to see functional outcome of this procedure.

\section{MATERIAL AND METHODS}

This is an observational prospective study carried out at two tertiary care centers at Nepal and Pakistan with similar protocol. Habitual and pathological recurrent dislocations were excluded. Recurrent dislocations of shoulder following initial traumatic anterior dislocation were included in this study. Twenty one patients were operated for recurrent traumatic anterior dislocation of shoulder between September 2000 to August 2007. They were followed up and evaluated for various periods. Among 21, 14 cases were operated in Bir Hospital, Kathmandu, Nepal and seven in Civil hospital, Nawabshah, Pakistan.

Approval and consent had been taken from the patients after explaining the procedure. Axillary approach was undertaken for the all lady patients (9) for cosmesis; Delto-pectoral approach was used for men (12). Cephalic vein served as a landmark for plane of dissection to separate and retract Deltoid and Pectoralis major on either side to expose the Coracoid process with its attached conjoint tendons of Coracobrachialis and short head of Biceps. Coracoid process then was osteotomised just distal to the insertion of Pectoralis minor without pre-drilling (Picture 1). It was reflected down to expose the Subscapularis muscle. This muscle was then split along its fibers at junction of its mid and lower third to

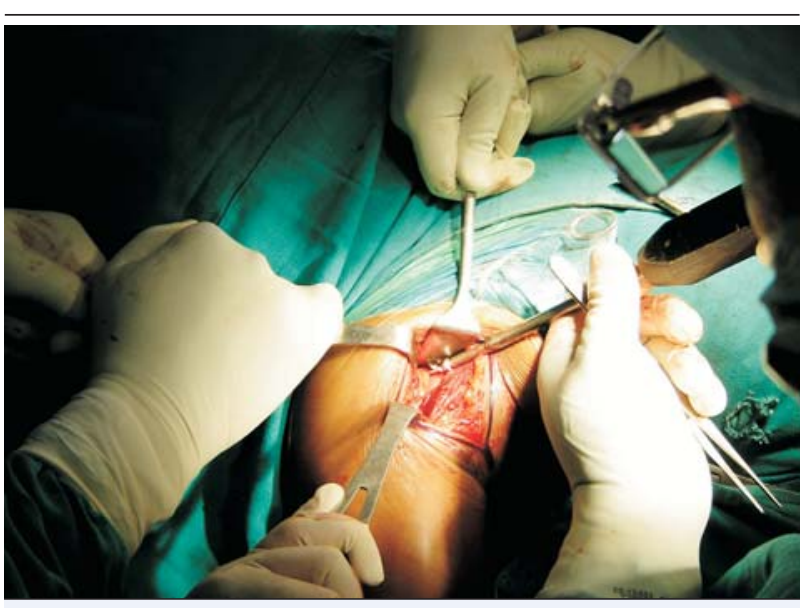

Picture 1. Osteotomised coracoid tip being screwed with Malleolar screw.

expose the joint capsule. The capsule was opened along the same line to inspect the joint cavity for any loose bodies, or any co-existing intraarticular pathology. While the joint lay open Glenoid margin was palpated to find and confirm the desired location of the screw placement. With this guide Scapular neck was drilled just below the transverse equator within one $\mathrm{cm}$ of the rim of Glenoid with a $3.2 \mathrm{~mm}$ drill bit. The joint capsule would then be closed. Next the coracoid process was also drilled with same drill bit. A Malleolar screw with a washer was used to fix the coracoid tip to the scapular neck through the Subscapularis split (Picture 2).

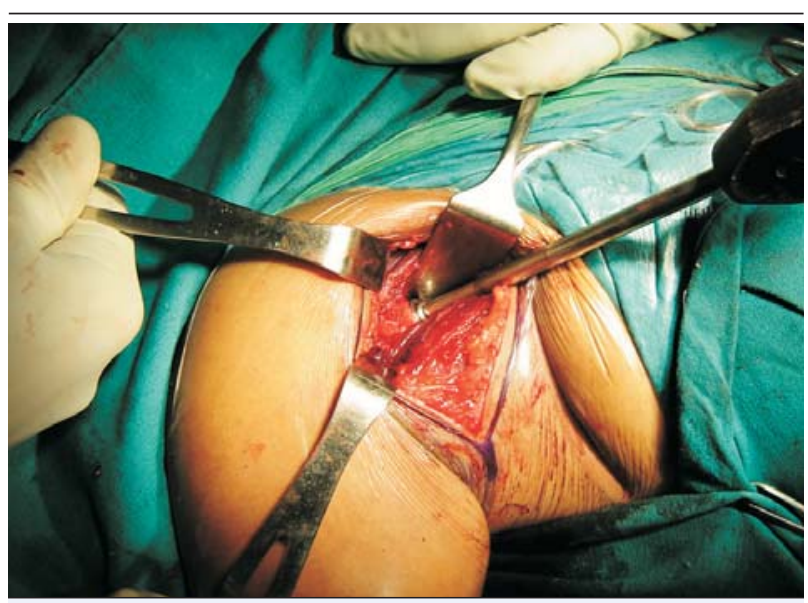

Picture 2. The Malleolar screw fixing the coracoids piece to the scapular neck.

Closure of the wound was affected by letting separated muscles fall back and stitching the subcutaneous tissue, skin over a suction drain. Postoperatively the shoulder was then immobilized in an immobilizer for one week. Then after an arm sling was worn for another four weeks. 
Then the sling was removed and pendulum exercise of the shoulder was begun. Active elbow flexion/extension was allowed only after three months to prevent pull out of the screw fixation. However passive flexion of the elbow was encouraged. Shoulder muscle strengthening and stretching exercises were started only after three months and continued for one-year (Picture 3). All activities including lifting weights were allowed after six months.

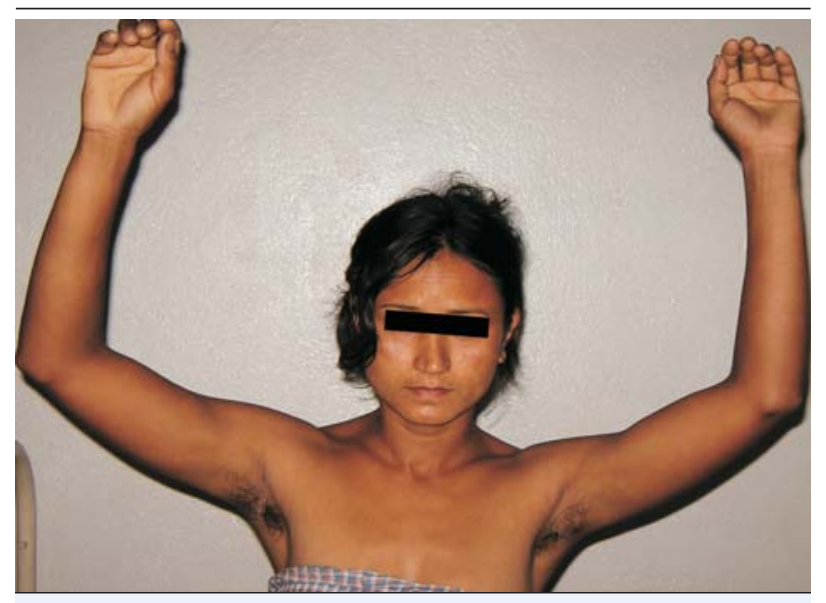

Picture 3. One patient with full range of shoulder motion after two year demonstrating it.

Follow up of all patients were carried out at one week for change of support, at two weeks for removal of stitches and after four weeks for removal of support and commencement of pendulum exercises. Further follow up was carried out at three months intervals for any problems. Antero-posterior and axillary view x-rays were taken at six weeks, three months and six months to see state of screw after the first postoperative check x-ray (Picture 4). Union of the coracoids with scapula is difficult to make out. However any pull out or loosening etc of the screw if any can be seen. Muscle power was measured for shoulder abductors, external rotators and internal rotators according to medical research council (MRC) grading and compared with the non-operated side. Range of motion was also noted in all directions. Function was assessed using the Rowe scoring system. ${ }^{7}$ It evaluated the overall success of the stabilizing procedure since half of the points were given for stability. Other points were given for range of movements and functional results.

Statistical calculation were done by using statistical package for social sciences (SPSS) version 11.5.

\section{RESULTS}

There were 12 men and 9 women. They were aged from 16 years to 49 years mean ages of 23.4 years. Eighteen patients were right handed and remaining 3 left handed. All dislocations were unilateral. There were 16 right sided and 5 left sided dislocations. Of those five left sided dislocations, only two patients were left-handed. Thus except these 2 patients all patient had dislocation on dominant hand side. Four patients, two men and two women, redislocated their shoulder even after Putti-Platt operation performed at other hospitals in Kathmandu.

At operation no intraarticular pathology nor any loose body was detected in any of the cases. There was no radiological evidence of loosening, migration or fracture of the coracoid screw nor resorption of the coracoid tip

Table 1. Rowe scoring at one year and two year follow-ups.

Rowe score at first year

\begin{tabular}{|c|c|c|c|c|c|}
\hline & & Frequency & Percent & Valid Percent & Cumulative Percent \\
\hline \multirow[t]{4}{*}{ Valid } & $75-89$ (Good) & 7 & 33.3 & 33.3 & 33.3 \\
\hline & 51-74 (fair) & 11 & 52.4 & 52.4 & 85.7 \\
\hline & $<50$ (poor) & 3 & 14.3 & 14.3 & 100.0 \\
\hline & Total & 21 & 100.0 & 100.0 & \\
\hline
\end{tabular}

Rowe score at second year

\begin{tabular}{|c|c|c|c|c|c|}
\hline & & Frequency & Percent & Valid Percent & Cumulative Percent \\
\hline \multirow[t]{4}{*}{ Valid } & $>90$ (Excellent) & 3 & 14.3 & 14.3 & 14.3 \\
\hline & $75-89$ (Good) & 15 & 71.4 & 71.4 & 85.7 \\
\hline & 51-74 (fair) & 3 & 14.3 & 14.3 & 100.0 \\
\hline & Total & 21 & 100.0 & 100.0 & \\
\hline
\end{tabular}




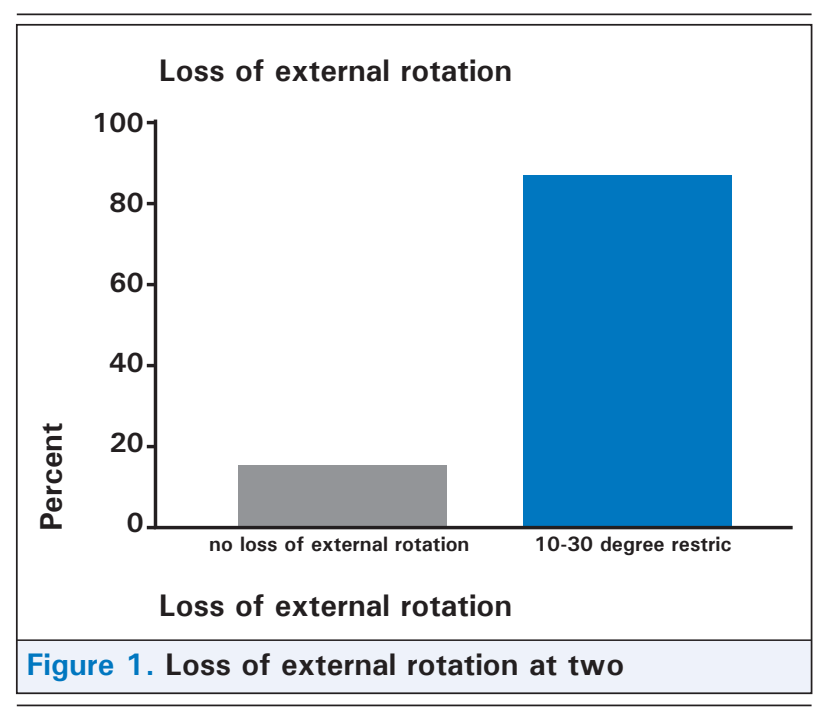

on subsequent check x-rays. Dislocation did not occur again in any of the case. At twelve months, 11 patients had fair Rowe score (51-74) and seven patients attained more than 75 (Good) Rowe scoring while at two years three had fair Rowe score, 15 had good Rowe score and three patients had excellent Rowe score (>90). Only three patients had poor score at one year while none had poor score at two year.

The mean loss of external rotation in operated shoulder at one year was 24.33 degrees which decreased to 21.2 degrees at the end of two years. There was no restriction of external rotation in three patients at both yearly followups while 18 patients had restriction in the range 1030 degrees.

Complications wise there were four superficial infections, one deep infection. All of them resolved with appropriate treatment with antibiotics and dressings. There were two cases of operation scar hypertrophy. Five coracoids were seen separated but not displaced even at the last follow up. They were asymptomatic and not interfering with the outcome of the surgery.

\section{DISCUSSION}

Recurrence of dislocation in shoulder reaches up to 80$90 \%$ in young adults and are disabling. As $97 \%$ of shoulder dislocations are anterior, recurrent dislocations are also usually anterior. No single pathoanatomy is responsible for it. Surgery is the treatment of choice. More than 150 operations and many modifications have been put forward for it but no single procedure is yet considered to be ideal. Although ability to detect specific pathology can lead a surgeon to correct it, choice of operation in practice is dependant upon preference of the surgeons and empirical in this part of world. Some how for fear of screw related technical difficulty and complications; Bristow procedure is not at all popular and is not performed usually in these two countries. However our experience with this procedure in comparision with other popular and simpler procedures in our practice had been superior.

Laterjet, Helfet, Mead were the pioneers in popularizing the coracoid transposition. ${ }^{8}$ The transferred conjoined tendon acts as a dynamic sling preventing forward and downward movement of the humeral head when arm is abducted. During abduction of the shoulder this transfer also prevents the lower half of the subscapularis muscle to slip superiorly, and is rather slipped inferiorly to supports the humeral head inferiorly. The coracoids piece also acts as a bone block anteriorly. Because of these effects this procedure has a good restraining to stop recurrence of the humeral head dislocation anteriorly regardless of pathology of recurrence. Thus this procedure is effective even after failure of other soft tissue procedure. In fact four such cases who had puttiplatt operation elsewhere underwent this procedure in this series and were cured finally of the recurrence.

Much has been written in the literature about this procedure with both mid-term and long term followups. ${ }^{9,10}$ All of them have shown satisfactory results with very few recurrences. But most of them uniformly had some degrees of limitation in external rotation of the shoulder. Few of the patients had bone and screw related complications. Singer, GS et al. reported 20 year follow up of this procedure in 14 cases. ${ }^{11}$ One patient had recurrence; 12 had restriction of external rotation; the functional outcome by Row scoring was satisfactory but there were radiological degenerative changes in 10 shoulders. In this series, the longest follow up was five years and shortest was two years. No one had any redislocation so far. Eighteen patients (85.7\%) had varying degrees of restriction in external rotation. Functional outcome based on Rowe scoring was excellent in $14.3 \%$, fair in $14.3 \%$ and good in $71.4 \%$ while none patients had poor Rowe score.

Hovelius et al reported a $16 \%$ incidence of screw migration, a $14 \%$ incidence of fracture at surgery of the coracoid tip and a $28 \%$ incidence of fibrous union of the transplanted coracoid. In our series we did not encountered such complications.

Subsequent $x$-rays did not reveal any screw pull outs although in five cases the coracoid piece could be seen 
separate from the scapular neck but undisplaced. In rest of the cases the coracoids process could be seen continuous with the scapular neck meaning union. Hence there were no screw related complications encountered in this series.

'Dislocation arthropathy' has been described by Samilson and Prieto. ${ }^{12}$ In 70 patients with recurrent dislocation, at from 1 to 50 years of follow-up, they found $59 \%$ incidence of arthropathy, but were unable to correlate the number of dislocations with the development or the severity of arthropathy. Posterior dislocations were more likely to lead to arthropathy than anterior dislocations, probably because of known delays in diagnosis and reduction after posterior dislocation.

We did not see any radiological features of degenerative changes in the shoulder joint on subsequent $x$-rays. May be our patients were not involved in contact sports or equivalent heavy physical activities and also our follow up were comparatively of shorter period.

\section{CONCLUSION}

Coracoid transposition following course of the conjoint tendon of short head of Biceps and Coraco-Brachialis provides mechanical block and dynamic support in positions of vulnerability thus providing good stability to the Gleno-Humeral joint. So it can be the procedure of choice for surgical treatment of recurrent traumatic anterior dislocation and also as a salvage surgery for failed cases from other types of procedures. The only shortcoming of this procedure was some limitation in external rotation.

\section{REFERENCE}

1. Hovelius L, Sandstrom BC, et al. Long-term results with the Bankart and Bristow-Latarjet procedures: recurrent shoulder instability and arthropathy. J Shoulder Elbow Surg 2001;10(5):445-52.

2. Helfet, AJ. Coracoid Transplantation for Recurring Dislocation of the Shoulder. J Bone and Joint Surg 1958;40.B(2):198-202.

3. Wildner $\mathrm{M}$, Terreri S, Reichelt $\mathrm{A}$. The X-ray appearance of the shoulder 10-23 years after the Eden-Hybbinette procedure. J Shoulder Elbow Surg 1993;Supp 16:51.

4. Barry TP, Lombardo SJ et al. The coracoid transfer for recurrent anterior instability of the shoulder in adolescents. J Bone Joint Surg [Am] 1985;67(3):383-7.

5. Lusardi DA, Wirth MA, Wurtz D, Rockwood CA Jr. Loss of external rotation following anterior capsulorraphy of the shoulder. J Bone Joint Surg [Am] 1993;75:1185-92.

6. Lombardo S J, Kerlan R K, Jobe F W, Carter V S, Blanzina ME, Shields CL, et al. The Modified Bristow Procedure for Recurrent
Dislocation of the Shoulder. J Bone and Joint Surg 1976;58A:256-261.

7. Rowe CR, Patel D, Southmayd WW. The Bankart procedure: a long- term end-result study. J Bone Joint Surg [Am] I 978:60A:1-16.

8. Terry S Canale. Campbell's Operative Orthopedics. 9th ed. Philadelphia: Mosby;1998.

9. Hovelius L. Anterior dislocation of the shoulder in teenagers and young adults. J Bone Joint Surg 1987;69-A:393.

10. Ferlic DC, DiGiovine NM. A long-term retrospective study of the modified Bristow procedure. Am J Sports Med. 1988; 16:469-74.

11. Singer GS, Kirkland PM, Emery RJH. Coracoid transposition for recurrent anterior instability of the shoulder. J Bone Joint Surg [Brit ] 1995;77-B:73-6.

12. Samilson RL, Prieto V. Dislocation arthropathy of the shoulder. J Bone Joint Surg [Am] 1983;65-A:456-60. 